\title{
Multidrug Resistant Acinetobacter Species Infection among Neonatal Sepsis
}

Mohamed Mamdouh Gaafar ${ }^{1}$, Azza Ali Khalil ${ }^{1}$, Ahmed Anwr Shaheen ${ }^{2}$, Eman Mohamed Kamel*1

Departments of ${ }^{1}$ Pediatrics and ${ }^{2}$ Microbiology and Immunology, Faculty of Medicine, Zagazig University, Egypt

*Corresponding author: Eman Mohamed Kamel, E-mail: whiteheart2013@yahoo.com

\begin{abstract}
Background: Neonatal septicemia is regarded as one of the leading causes of mortality as well as morbidity globally. There is emerging evidence that multidrug resistant Acinetobacter baumannii (MDRAB) and mortality are linked in the scientific literature.

Objective: It was the goal of this work to improve the prognosis of neonates with Acinetobacter species through early detection of infection and risk factors associated with increased mortality and effective management.

Patients and Methods: Our study was done on 60 neonates who were suspected to having sepsis at Zagazig University Hospitals, Pediatric Department. All neonatal blood samples were taken aseptically and the bacteria that caused septicemia were identified. Acinetobacter species were identified. Drug sensitivity tests were performed on a variety of risk variables.

Results: Only nine patients had Acinetobacter infection (15\% of all patients) and two thirds of them had multi drug resistance (resistant for $\geq 3$ antimicrobials). Gestational age more than or equal 36 weeks was protective factor against getting infection with MDR-Acinetobacter among the studied patients. Acinetobacter was most sensitive to ciprofloxacin and tigecycline antibiotic, while it was most resistant to sulphamethoxazole/trimethoprim antibiotics Conclusion: Neonatal MDR Acinetobacter septicemia is on the rise, and it's connected with high morbidity as well as mortality rates. There must be an infection control policy in place at every neonatal intensive care unit (NICU) in order to control Acinetobacter infection and enhance outcomes.
\end{abstract}

Keywords: Multidrug Resistant Acinetobacter, Neonatal sepsis.

\section{INTRODUCTION}

If a newborn child is diagnosed with sepsis, they have a bloodstream infection that is less than 28 days old. Neonatal morbidity as well as mortality continue to be a major problem in low and middle-income countries. Based on the period of manifestation after birth, neonatal sepsis can be separated into two types: early-onset sepsis (EOS) in addition to late-onset sepsis (LOS). Neonatal sepsis occurring within the first 72 hours of life (some specialists use 7 days) is referred to as EOS, while sepsis happening at or after the last 72 hours of life is referred to as LOS ${ }^{(\mathbf{1})}$.

In neonatal intensive care units (NICUs), identifying and treating sepsis is a major issue for neonatologists. Nonspecific symptoms and indications make it difficult to make a clinical diagnosis. As a result, laboratory testing is a time-consuming process. When a patient is suspected of having sepsis, it is imperative to begin empirical antibiotic therapy immediately. As a result, the number of treatment alternatives is decreasing and the time it takes for an effective treatment is increasing (2).

Additionally, Acinetobacter spp. are becoming increasingly important as a possible pathogen in neonatal septicemia due to their frequent isolation, antibiotic resistance, and greater fatality rates compared to Klebsiella and Salmonella spp. ${ }^{(3)}$.

Acinetobacter spp. is generally considered to be a low-virulence organism, but conditions like immunocompromised status, critical illness, prematurity, broad-spectrum antibiotic therapy, endotracheal intubation, low birth weight, intravascular catheterization, as well as parenteral nutrition, are known risk factors for Acinetobacter spp. infection induced sepsis ${ }^{(4)}$. Mortality has been linked to MDR Acinetobacter baumannii (MDRAB) in the recent years literatures ${ }^{(\mathbf{5})}$.

Neonatal Acinetobacter infection is associated with a significant death rate, however there is a lack of research pinpointing the elements that contribute to this ${ }^{(6)}$. Bacterial pathogen Acinetobacter baumannii is an opportunistic hospital-acquired infection-causing pleomorphic, nonmotile Gramnegative bacillus, as well as aerobic organisms ${ }^{(7)}$.

Infections such as meningitis, wound infections, urinary tract infections, bacteremia and lower respiratory tract can be caused by it. Antibiotic-resistant strains of Acinetobacter baumannii have spread around the globe in a short period of time. Bacteria resistant to three or more of the following types of antimicrobials are known as multidrug resistant Acinetobacter baumannii (MDRAB): aminoglycosides, penicillins, fluoroquinolones, antipseudomonal, carbapenems, cephalosporins, beta-lactamase inhibitors, polymyxins, and tetracycline, folate pathway inhibitors. Pandrugresistant to qualify as Acinetobacter baumannii, one must be resistant to all of these categories ${ }^{(8)}$.

It was the goal of this work to improve the prognosis of neonates with Acinetobacter species through early detection of infection and risk factors associated with increased mortality and effective management. 


\section{PATIENTS AND METHODS}

Sixty newborns hospitalized at Zagazig University Hospitals, in the neonatal intensive care unit between June 2019 and March 2020 served as the subjects for this cross-sectional trial.

\section{Ethical considerations:}

As long as all parents of participants signed informed consent forms and submitted them to Zagazig University's Research Ethics Committee, the study was allowed (ZU-IRB\#6270). We followed the World Medical Association's ethical code for human experimentation, the Helsinki Declaration.

Inclusion criteria: All established newborns with sepsis-related symptoms, and signs at the time of admission or who contracted sepsis during their hospital stay.

Exclusion criteria: Neonates with multiple congenital anomalies, genetic syndromes, bleeding disorders.

This is what all of the participants in this research had to go through:

History: The patient's age, sex, gender, maternal risk factors, gestational age, prenatal, natal history were all recorded in a thorough medical history.

Clinical examination: Weight of a newborn, suckling and Moro reflexes (Moro) were performed on the newborns as well as vital parameters such as heart rate and respiration rate, spotting the early indications of sepsis: Restlessness, sleepiness, pallor, and mottled skin characterise the infant's condition. A fluctuation in temperature, either hyperthermia or hypothermia problem with the respiratory system.

Laboratory evaluation: Blood samples were taken at the time of sepsis suspicion. Skin was rubbed with antiseptic and $4 \mathrm{~cm}$ of blood were taken: $1 \mathrm{~cm}$ of blood was collected in a test tube containing $20 \mathrm{mcg}$ of EDETA for CBC, $2 \mathrm{~cm}$ of blood were collected in a plain test tube for CRP and serum creatinine, $1 \mathrm{~cm}$ of blood was injected into culture bottle.

Complete Blood Count: Analysed by Sysmex 21-kx cell counter for hemoglobin level, red blood cell count, RDW, hematocrit value, platelet count and white blood cell (WBC) count (Total and differential). Results of CBC were interpreted using hematological scoring system by Rodwell et al. ${ }^{(9)}$.

Quantitative C- reactive protein (CRP): $1 \mathrm{~cm}$ of blood was taken, blood was collected in a plain test tube, left to clot, then centrifuged for 10 minutes at $1500 \mathrm{rpm}$, Turbox plus was used to separate and analyze serum. Above $6 \mathrm{mg} / \mathrm{l}$, results were deemed positive.

Blood culture: For the first three days, the blood cultures were incubated aerobically at $37^{\circ} \mathrm{C}$ and monitored daily for the presence of observable microbial growth via one of the following: air bubbles (gas generation), hemolysis, or broth coagulation.

Simultaneously, subcultures were established on enriched and selective medium such as blood, chocolate, MacConkey, and mannitol salt agar plates for three consecutive days and then tested for growth after 24-48 hours of incubation. The same procedure was continued until the blood culture was determined to be microorganism-free on the seventh day. Standard microbiological techniques were used to identify isolated organisms, including Gram staining, colony features, and biochemical properties ${ }^{(\mathbf{1 0})}$.

\section{Antibiotic sensitivity pattern:}

Muller-Hinton agar (Oxoid): Beef dehydrated infusion: $300 \mathrm{gm}$, casein hydrolysate: $17.5 \mathrm{gm}$, starch: 1.5 gm and agar: $17 \mathrm{gm}(\mathrm{pH}: 7.3 \pm 0.1)$. Thirty-eight grams were suspended in 1 liter of distilled water and boiled to dissolve completely. After sterilization with an autoclave at $121^{\circ} \mathrm{C}$ for 15 minutes, the medium was dispensed in sterile Petri dishes.

\section{Methods:}

Disk diffusion was used to detect antibiotic resistance among the isolates. Simply four to five isolated colonies with comparable morphology from each isolate were looped, injected into $5 \mathrm{ml}$ sterile nutrient broth, and incubated at $35^{\circ} \mathrm{C}$ for $2-5$ hours until turbidity was produced. The turbidity was then corrected to 0.5 McFarland standard using saline. Inocula were administered to Muller-Hinton agar plates using sterile swabs from these broth suspensions ${ }^{(11)}$.

After blood culture the patients were classified into 2 groups: Group 1: Included 9 patients and they were Acinetobacter +ve, and Group 2: Included 51 patients and they were Acinetobacter -ve.

After antibiotic sensitivity, the patients of group 1 were further subdivided into multidrug resistant Acinetobacter (MDR) and they were 6 patients and 3 patients had usual Acinetobacter infection (i.e. not MDR)

\section{Statistical analysis}

The collected data were coded, processed and analyzed using the SPSS (Statistical Package for Social Sciences) version 22 for Windows ${ }^{\circledR}$ (IBM SPSS Inc, Chicago, IL, USA). Quantitative data were presented as means + standard deviation (SD), median, and range. The independent $\mathrm{t}$-test $(\mathrm{t})$ and the Mann-Whitney (MW) test were employed to compare parametric and non-parametric quantitative data, respectively, in the analysis of the differences between the groups. Qualitative values were presented as numbers and proportions, which were compared using the Chi-square test $\left(\mathrm{X}^{2}\right)$ or Fisher's exact test. $\mathrm{P}$ value $<0.05$ was considered statistically significant.

\section{RESULTS}

The presence of Acinetobacter didn't have a statistically significant correlation with either gender, age, body weight, Apgar score, or 1 or 5 minutes of time. There was statistically significant relation between presence of Acinetobacter and gestational age (at 36 weeks) of the studied patients (Table 1). 
Table (1): Patients' demographic and clinical information was gathered for the study

\begin{tabular}{|c|c|c|c|}
\hline Parameter & Group 1, N=9 (\%) & Group 2, N=51 (\%) & $\mathbf{p}$ \\
\hline $\begin{array}{l}\text { Gender: } \\
\text { Female } \\
\text { Male }\end{array}$ & $\begin{array}{l}6(66.7) \\
3(33.3)\end{array}$ & $\begin{array}{l}23(45.1) \\
28(54.9)\end{array}$ & 0.292 \\
\hline $\begin{array}{l}\text { Gestational age: } \\
\text { Mean } \pm \text { SD } \\
<36 \text { weeks } \\
\geq 36 \text { weeks. }\end{array}$ & $\begin{array}{c}33.889 \pm 2.713 \\
8(88.9) \\
1(11.1) \\
\end{array}$ & $\begin{array}{c}35.569 \pm 2.427 \\
22(43.1) \\
29(56.9)\end{array}$ & $\begin{array}{c}0.065 \\
0.026 *\end{array}$ \\
\hline Age (days): Median (range) & $10(5-28)$ & $11(5-23)$ & 0.236 \\
\hline Birth weight (kg):Mean \pm SD & $2.411 \pm 0.936$ & $2.453 \pm 0.675$ & 0.871 \\
\hline APGAR at 1 minute: Mean \pm SD & $6.667 \pm 1.581$ & $6.823 \pm 1.178$ & 0.728 \\
\hline APGAR at 5 minutes: Mean \pm SD & $8.778 \pm 1.302$ & $8.941 \pm 1.047$ & 0.679 \\
\hline
\end{tabular}

*: Statistically significant, Bacteria was most sensitive to ceftazidime antibiotic, while it was most resistant to the combination of sulphamethoxazole and trimethoprim antibiotics (Table 2).

Table (2): Distribution of the studied patients according to resistant patterns to various antibiotics

\begin{tabular}{|c|c|c|c|}
\hline \multirow{2}{*}{ Antimicrobials } & Sensitive & Intermediate & Resistan \\
\hline & $\mathbf{N}(\%)$ & $\mathbf{N}(\%)$ & $\mathbf{N}(\%)$ \\
\hline Levofloxacin & $20(33.3)$ & $7(11.7)$ & $7(11.7)$ \\
\hline Ceftazidime & $50(83.3)$ & $4(6.7)$ & $6(10)$ \\
\hline Ticarcillin & $0(0)$ & $0(0)$ & $3(5)$ \\
\hline Cefipim & $2(3.3)$ & $0(0)$ & $5(8.3)$ \\
\hline Cefotaxime & $0(0)$ & $0(0)$ & $13(21.7)$ \\
\hline Cefixim & $0(0)$ & $0(0)$ & $11(18.3)$ \\
\hline Cefotriaxone & $2(3.3)$ & $0(0)$ & $14(23.3)$ \\
\hline Amoxacillin & $0(0)$ & $1(1.7)$ & $29(48.3)$ \\
\hline Ampicillin & $0(0)$ & $0(0)$ & $23(38.3)$ \\
\hline Aztronem & $1(1.7)$ & $0(0)$ & $11(18.3)$ \\
\hline Vancomycin & $16(26.7)$ & $0(0)$ & $6(10)$ \\
\hline Mecicillin & $2(3.3)$ & $0(0)$ & $2(3.3)$ \\
\hline Spiramycin & $2(3.3)$ & $0(0)$ & $0(0)$ \\
\hline Azithromycin & $2(3.3)$ & $0(0)$ & $5(8.3)$ \\
\hline Clindamycin & $11(18.3)$ & $0(0)$ & $10(16.7)$ \\
\hline Tetracycline & $8(13.3)$ & $0(0)$ & $9(15)$ \\
\hline Erythromycin & $0(0)$ & $0(0)$ & $9(15)$ \\
\hline Ciprofloxacin & $12(20)$ & $2(3.3)$ & $15(25)$ \\
\hline Tobramycin & $3(5)$ & $0(0)$ & $3(5)$ \\
\hline Tigercycline & $20(33.3)$ & $1(1.7)$ & $0(0)$ \\
\hline Amikin & $5(8.3)$ & $0(0)$ & $14(23.3)$ \\
\hline Peflacin & $3(5)$ & $2(3.3)$ & $0(0)$ \\
\hline Sulphamethaxole-trimethoprim & $6(10)$ & $1(1.7)$ & $28(46.7)$ \\
\hline Meronem & $3(5)$ & $1(1.7)$ & $14(23.3)$ \\
\hline Cefadroxil & $0(0)$ & $0(0)$ & $3(5)$ \\
\hline Mecicillin & $0(0)$ & $0(0)$ & $3(5)$ \\
\hline Linezolid & $14(23.3)$ & $0(0)$ & $0(0)$ \\
\hline Piperacillin & $0(0)$ & $0(0)$ & $3(5)$ \\
\hline Colistin & $5(8.3)$ & $2(3.30$ & $0(0)$ \\
\hline Metronidazole & $0(0)$ & $0(0)$ & $3(5)$ \\
\hline Moxifloxacin & $3(5)$ & $2(3.3)$ & $5(8.3)$ \\
\hline Oxacillin & $0(0)$ & $0(0)$ & $6(10)$ \\
\hline Rifampicin & $0(0)$ & $0(0)$ & $3(5)$ \\
\hline Fortum & $1(1.7)$ & $0(0)$ & $0(0)$ \\
\hline Norfloxacin & $1(1.7)$ & $0(0)$ & $0(0)$ \\
\hline Polymyxin & $2(3.3)$ & $3(5)$ & $0(0)$ \\
\hline Chloramphenicol & $1(1.7)$ & $1(1.7)$ & $0(0)$ \\
\hline Ceftazidime/avibactam & $2(3.3)$ & $0(0)$ & $0(0)$ \\
\hline
\end{tabular}

K. pneumonia represented the commonest organism (21.7\%) followed by Acinetobacter and Staph hominis (15\%) then pseudomonas (13.3\%) (Figure 1). 


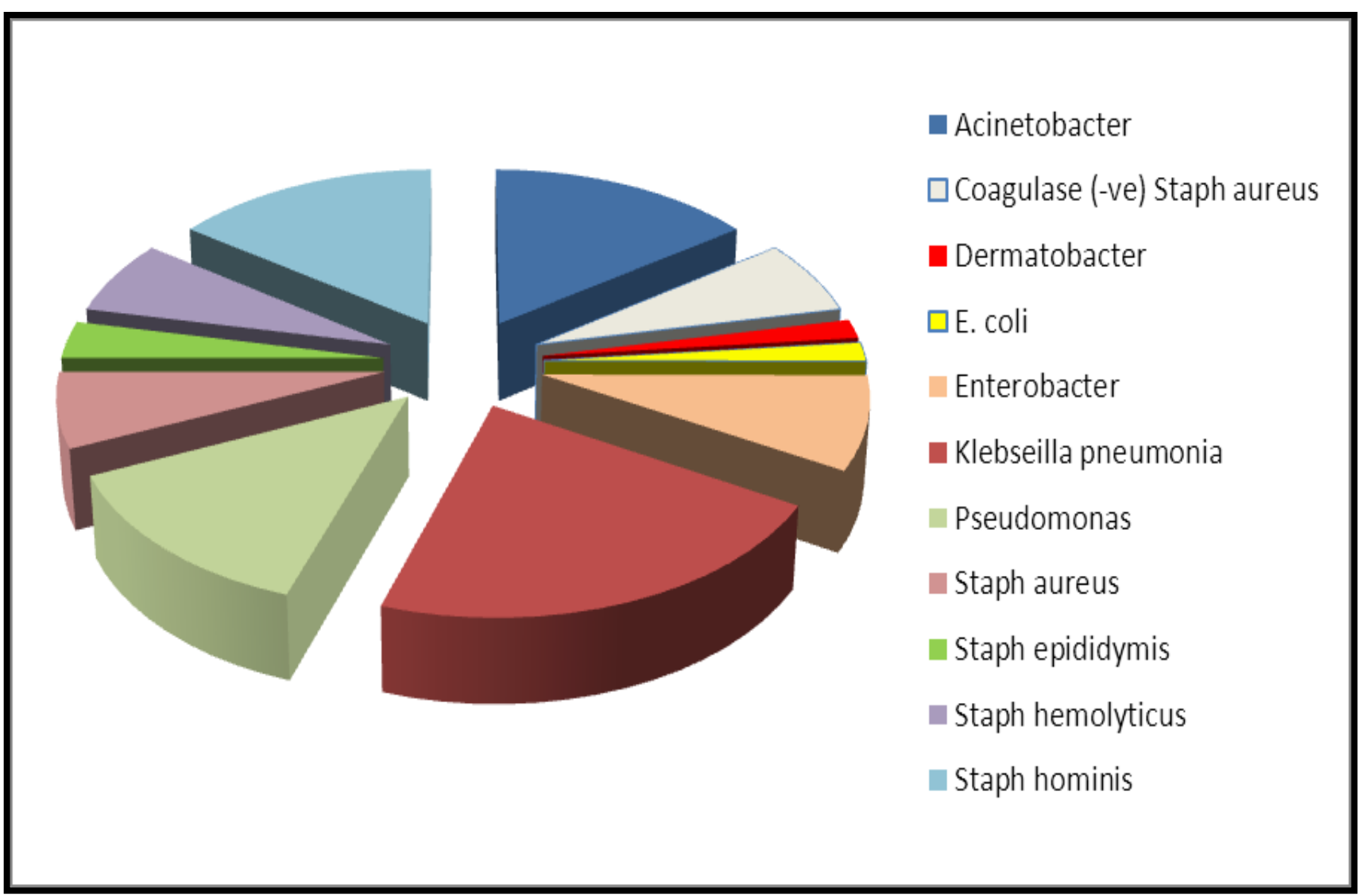

Fig. (1): Pie chart showing distribution of patients according to culture result

Acinetobacter was most sensitive to ciprofloxacin and tigecycline antibiotic, while it was most resistant to sulphamethoxazole/trimethoprim antibiotics (Table 3).

Table (3): Distribution of Acinetobacter according to culture and sensitivity to various antibiotic

\begin{tabular}{|c|c|c|c|}
\hline \multirow{3}{*}{ Antimicrobials } & \multicolumn{3}{|c|}{ Acinetobacter $(\mathrm{n}=9)$} \\
\hline & Sensitive & Intermediate & Resistant \\
\hline & $\mathbf{N}(\%)$ & $\mathrm{N}(\%)$ & $\mathbf{N}(\%)$ \\
\hline Levofloxacin & $1(11.1)$ & $0(0)$ & $0(0)$ \\
\hline Cefipim & $0(0)$ & $0(0)$ & $1(11.1)$ \\
\hline Cefotaxim & $0(0)$ & $0(0)$ & $1(11.1)$ \\
\hline Cefixim & $0(0)$ & $0(0)$ & $3(33.3)$ \\
\hline Cefotriaxone & $0(0)$ & $0(0)$ & $1(11.1)$ \\
\hline Amoxacillin & $0(0)$ & $0(0)$ & $6(66.7)$ \\
\hline Ampicillin & $0(0)$ & $0(0)$ & $6(66.7)$ \\
\hline Aztronem & $0(0)$ & $0(0)$ & $2(22.2)$ \\
\hline Vancomycin & $0(0)$ & $0(0)$ & $1(11.1)$ \\
\hline Tetracycline & $1(11.1)$ & $0(0)$ & $0(0)$ \\
\hline Ciprofloxacin & $4(44.4)$ & $1(11.1)$ & $1(11.1)$ \\
\hline Tobramycin & $3(33.3)$ & $0(0)$ & $2(22.2)$ \\
\hline Tigecycline & $4(44.4)$ & $1(11.1)$ & $0(0)$ \\
\hline Amikin & $3(33.1)$ & $0(0)$ & $1(11.1)$ \\
\hline Peflacin & $3(33.3)$ & $0(0)$ & $0(0)$ \\
\hline Sulphamethaxole-trimethoprim & $0(0)$ & $0(0)$ & $8(88.9)$ \\
\hline Meronem & $1(11.1)$ & $0(0)$ & $2(33.3)$ \\
\hline Moxifloxacin & $0(0)$ & $0(0)$ & $2(22.2)$ \\
\hline
\end{tabular}

Only nine patients had Acinetobacter infection (15\% of all patients) and two thirds of them had multi drug resistance (resistant for $\geq 3$ antimicrobials) (Table 4). 
Table (4): Distribution of patients according to presence of Acinetobacter

\begin{tabular}{|l|c|c|}
\hline \multicolumn{1}{|c|}{ Acinetobacter } & $\mathbf{N = 6 0}$ & \% \\
\hline Yes & 9 & 15 \\
No & 51 & 85 \\
\hline MDR Acinetobacter: & $\mathbf{N = 9}$ & \\
No & 3 & 33.3 \\
Yes & 6 & 66.7 \\
\hline
\end{tabular}

There was statistically non-significant relation between presence of MDR Acinetobacter and either gender, age, body weight, Apgar score and 1 or 5 minutes. There was statistically significant relation between presence of MDR Acinetobacter and gestational age of the studied patients (Table 5).

Table (5): Relation between presence of MDR Acinetobacter and both demographic and clinical data of the studied patients

\begin{tabular}{|c|c|c|c|}
\hline \multirow[b]{2}{*}{ Parameter } & \multicolumn{2}{|c|}{ MDR Acinetobacter } & \multirow[b]{2}{*}{$\mathbf{p}$} \\
\hline & $\begin{array}{c}\text { Present } \\
\mathrm{N}=6(\%)\end{array}$ & $\begin{array}{c}\text { Absent } \\
\mathrm{N}=54(\%)\end{array}$ & \\
\hline $\begin{array}{l}\text { Gender: } \\
\text { Female } \\
\text { Male } \\
\end{array}$ & $\begin{array}{l}4(66.7) \\
2(33.3) \\
\end{array}$ & $\begin{array}{l}25(46.3) \\
29(53.7) \\
\end{array}$ & 0.417 \\
\hline $\begin{array}{l}\text { Gestational age: } \\
\text { Mean } \pm \text { SD } \\
<36 \text { weeks } \\
\geq 36 \text { weeks. } \\
\end{array}$ & $\begin{array}{c}34 \pm 1.095 \\
6(100) \\
0(0)\end{array}$ & $\begin{array}{c}35.463 \pm 2.597 \\
24(44.4) \\
30(55.6)\end{array}$ & $\begin{array}{r}0.024^{*} \\
0.02 *\end{array}$ \\
\hline $\begin{array}{l}\text { Age (days): } \\
\text { Median (range) }\end{array}$ & $9.5(5-28)$ & $11(5-23)$ & 0.38 \\
\hline $\begin{array}{l}\text { Weight }(\mathbf{k g}): \\
\text { Mean } \pm \text { SD }\end{array}$ & $2.483 \pm 0.847$ & $2.443 \pm 0.703$ & 0.896 \\
\hline $\begin{array}{l}\text { APGAR at } 1 \text { minute: } \\
\text { Mean } \pm \text { SD }\end{array}$ & $6.667 \pm 1.211$ & $6.815 \pm 1.245$ & 0.783 \\
\hline $\begin{array}{l}\text { APGAR at } 5 \text { minutes: } \\
\text { Mean } \pm \text { SD }\end{array}$ & $9 \pm 0.894$ & $8.907 \pm 1.103$ & 0.844 \\
\hline
\end{tabular}

*: Statistically significant

Gestational age more than or equal to 36 weeks was protective factor against getting infection with MDRAcinetobacter among the studied patients (Table 6).

Table (6): Multivariate regression analysis of factors associated with presence of MDR-Acinetobacter infection among the studied patients

\begin{tabular}{|c|c|c|c|c|c|}
\hline \multirow{2}{*}{ Parameter } & \multirow{2}{*}{$\boldsymbol{\beta}$} & \multirow{2}{*}{$\mathbf{p}$} & \multirow{2}{*}{ AOR } & \multicolumn{2}{|c|}{ 95\% C.I. } \\
\cline { 5 - 6 } & & & & Lower & Upper \\
\hline GA $(\geq 36$ week $)$ & -19.82 & 0.998 & 0 & 0 & 0 \\
\hline
\end{tabular}

There was statistically non-significant relation between presence of MDR Acinetobacter and patient outcome. MDR Acinetobacter non-significantly increased mortality risk by 3.19 fold (Table 7).

Table (7): Relation between presence of MDR Acinetobacter and patient outcome

\begin{tabular}{|c|c|c|c|c|}
\hline \multirow[b]{2}{*}{ MDR Acinetobacter } & \multicolumn{2}{|c|}{ Outcome } & \multirow[b]{2}{*}{$\mathbf{p}$} & \multirow[b]{2}{*}{$\operatorname{COR}(95 \% \mathrm{CI})$} \\
\hline & $\begin{array}{c}\text { Died } \\
\mathrm{N}=6(\%)\end{array}$ & $\begin{array}{l}\text { Improved } \\
\mathrm{N}=54(\%)\end{array}$ & & \\
\hline $\begin{array}{l}\text { Present } \\
\text { Absent }\end{array}$ & $\begin{array}{c}3(15.8) \\
16(84.2)\end{array}$ & $\begin{array}{c}3(7.3) \\
51(92.7)\end{array}$ & 0.37 & $3.19(0.58-17.38)$ \\
\hline
\end{tabular}




\section{DISCUSSION}

Acinetobacter species are oxidase-negative, catalase-positive, and Gram-negative coccobacilli that were first discovered in 1911 and have since been studied extensively. Microorganisms of the Acinetobacter genus can be found in a variety of natural habitats such as soil and water as well as in food and sewage. Non-fermentative organisms have emerged as important nosocomial infections in the hospital environment and have been responsible for occasional outbreaks ${ }^{(\mathbf{1 2})}$.

Nearly 80 percent of all reported Acinetobacter infections are caused by the Acinetobacter baumannii species, according to the Centers for Disease Control and Prevention (CDC). In intensive care units, A. baumannii is a leading source of healthcare-associated infections (HAIs) ${ }^{(13)}$.

Acinetobacter infection has been linked to prior antibiotic usage, prolonged hospitalization, high colonization pressure, and enteral feeding. More recently, Acinetobacter has become increasingly recognized as an important healthcare-associated MDR pathogen and its rate of isolation has increased over the past two decades worldwide ranging from 26.5 percent to 91 percent in immunocompromised patients ${ }^{(14)}$.

This study showed with gestational age lower than 36 weeks, actinobacteria prevalence was significantly higher in females than males. and this was supported by Keskar $\boldsymbol{e t}$ al. ${ }^{(15)}$ who revealed that Acinetobacter cases are more common in females, babies under $1500 \mathrm{~g}$ and under 30 weeks gestation than non-acinetobacter cases. Acinetobacter cases were less common among males, newborns with a birth weight of more than $1500 \mathrm{~g}$, gestational ages of more than 30 weeks, and normal deliveries, compared to non-Acinetobacter cases. However, in the study of Nazir, ${ }^{(16)}$ they reported that the male-to-female ratio was 2:1 among the 357 participants in the research. In 38 (77.5 percent) of the instances, the gestational age was 36 weeks and the low-birth-weight babies were 40 (81.6 percent).

Current study showed that K. pneumonia represented the commonest organism (21.7\%) followed by Acinetobacter and Staph. hominis (15\%) then pseudomonas (13.3\%). Our results were supported by study of Hannan $\boldsymbol{e t}$ al. ${ }^{(17)}$ as they demonstrated that K. pneumoniae $(n=40)$ and Coagulase-negative staphylococci (CoNS) $(\mathrm{n}=11)$ were the most common isolates. Akter et al. ${ }^{(18)}$ found that organisms isolated from the blood of the newborn are Acinetobacter spp. 32 (34.48\%), followed by Pseudomonas spp. 19 (21.83\%), CoNS 16 (18.39\%), Klebsiella and Moraxella spp. 6 (6.89\%) respectively, Staph. aureus 4 (5.97\%) Enterobacter spp. 3 (3.44\%), Esch. coli 2 (2.29\%) and Enterococci spp. 1(1.49\%). However, Awad et al. ${ }^{(19)}$ revealed that E. coli was the most common pathogen found in both early- and late- onset sepsis (41.2 percent and 24.5 percent), respectively.

The present study showed that Acinetobacter was most resistant to sulphamethoxazoletrimethoprim followed by amoxicillin, ampicillin and resistant to meronem was $33.3 \%$. Hannan et al. ${ }^{(17)}$ demonstrated that and over 90 percent of Gramnegative isolates were resistant to -lactams, coamoxiclav, and amikacin. Penicillin, macrolides, ciprofloxacin and co-trimoxazole were all shown to be ineffective against more than half of the Grampositive isolates. This was in agreement with our study as amoxicillin and sulpha co-trimoxazole had the highest resistance collectively. Nazir ${ }^{(16)}$ revealed that 95.9\% of the isolates were MDR, meaning they were resistant to, fluoroquinolones, penicillin, aminoglycosides as well as and cephalosporins (including inhibitor combinations). Despite this, all of the strains were responsive to colistin. MDR was found in 66.7 percent of the samples in our study. This difference may be due to the relatively appropriate neonatal care and infection control in our NICU, proper hand wash of medical staff, proper sterilization of instrumental devices and use of disinfection.

The current study showed that only nine patients had Acinetobacter infection (15\% of all patients) and two thirds of them had multi drug resistance (resistant for $\geq 3$ antimicrobials). About $32 \%$ of patients died by the end of the study. Sepsis accounted for $31.6 \%$ of mortality. Furthermore, Hsu et al. (20) demonstrated that in total, 37 cases of A baumannii bacteremia were found. Multidrugresistant isolate was found in only two neonates (5.4 percent), although overall mortality was 8.1 percent. Those with A baumannii bacteremia exhibited a high rate of recurrent LOS and a considerably greater rate of prolonged hospitalisation compared to patients with E coli and Klebsiella bacteremia (OR, 2.25; 95\% CI: 1.04-4.88, $\mathrm{P} 1 / 4.039)$. This may be due to it is more common in low birth weight (LBW) with high-rate central venous catheter (CVC) and total parenteral nutrition (TPN).

In the study in our hands, there was statistically non-significant relation between presence of MDR Acinetobacter and patient outcome. MDR Acinetobacter non-significantly increased mortality risk by 3.19-fold. This may be due to difference endemic pattern of organism. Our results were supported by study of Yusef $\boldsymbol{e t}$ al. ${ }^{(\mathbf{2 1 )})}$ as they reported that during the study period, the mortality rate for MDRAB infection in the NICU was 20.34 percent.

\section{CONCLUSION}

Neonatal septicemia is a very concerning health problem, healthcare environment has a very important role in developing septicemia among neonates. MDR is becoming increasingly common for neonatal patients to develop Acinetobacter septicemia, which has a high death and morbidity rate. 
Consequently, each neonatal intensive care unit must have an efficient infection control programs in place in order to control Acinetobacter infection and enhance patient outcomes. There was statistically significant relation between presence of Acinetobacter and gestational age (at 36 weeks) of the studied patients. K. pneumonia represented the commonest organism (21.7\%) followed by Acinetobacter and Staph. hominis (15\%) then pseudomonas (13.3\%). Acinetobacter was most sensitive to ciprofloxacin and tigecycline antibiotics, while it was most resistant to sulphamethoxazole/trimethoprim antibiotics. Gestational age more than or equal 36 weeks was protective factor against getting infection with MDRAcinetobacter among the studied patients.

Financial support and sponsorship: Nil.

Conflict of interest: Nil.

\section{REFERENCES}

1. Singh M, Alsaleem M, Gray C (2021): Neonatal sepsis.

StatPearls.https://www.ncbi.nlm.nih.gov/books/NB K531478/

2. Patel S, Saiman L (2010): Antibiotic resistance in neonatal intensive care unit pathogens: mechanisms, clinical impact, and prevention including antibiotic stewardship. Clinics in Perinatology, 37(3): 547-563.

3. Jeyamurugan T, Ragulganesh R, Sucilathangam G et al. (2012): Acinetobacter spp.: An Emerging Pathogen in Neonatal Septicaemia. JCDR., 6(5): 805806.

4. Wei H, Hsu Y, Lin $H$ et al. (2015): Multidrugresistant Acinetobacter baumannii infection among neonates in a neonatal intensive care unit at a medical center in central Taiwan. Journal of Microbiology, Immunology and Infection, 48(5): 531-539.

5. Saleem A, Ahmed I, Mir F et al. (2010): Panresistant Acinetobacter infection in neonates in Karachi, Pakistan. J Infect Dev Ctries., 4: 30-37.

6. Viswanathan R, Singh A, Basu S (2014): Multidrug-resistant, non-fermenting, gram-negative bacilli in neonatal sepsis in Kolkata, India: a 4-year study. Paediatr Int Child Health, 34(1):56-9.

7. Howard A, O'Donoghue M, Feeney A et al. (2012): Acinetobacter baumannii: an emerging opportunistic pathogen. Virulence, 3: 243-250.

8. Magiorakos A, Srinivasan A, Carey R et al. (2012): Multidrug-resistant, extensively drug-resistant and pandrug-resistant bacteria: an international expert proposal for interim standard definitions for acquired resistance. Clin Microbiol Infect., 18: 268-81.

9. Rodwell R, Leslie A, Tudehope D (1988): Early diagnosis of neonatal sepsis using a hematologic scoring system. The Journal of Pediatrics, 112(5): 761-767.

10. Patel D, Nimbalkar A, Sethi A et al. (2014): Blood culture isolates in neonatal sepsis and their sensitivity in Anand district of India. The Indian Journal of Pediatrics, 81(8): 785-790.

11. Jonasson E, Matuschek E, Kahlmeter G (2020): The EUCAST rapid disc diffusion method for antimicrobial susceptibility testing directly from positive blood culture bottles. Journal of Antimicrobial Chemotherapy, 75(4): 968-978.

12. Paolucci M, Landini M, Sambri V (2012): How can the microbiologist help in diagnosing neonatal sepsis?. International Journal of Pediatrics, 12: 26-31.

13. Sinha N, Agarwal J, Srivastava S et al. (2013). Analysis of carbapenem-resistant Acinetobacter from a tertiary care setting in North India. Indian $\mathbf{J}$ Med Microbiol., 31: 60-3.

14. Punpanich W, Nithitamsakun N, Treeratweeraphong $\mathrm{V}$ et al. (2012): Risk factors for carbapenem non-susceptibility and mortality in Acinetobacter baumannii bacteremia in children. Int J Infect Dis., 16: 811-5.

15. Keskar U, Parekh A, Behera M (2016): Multidrugresistant Acinetobacter species infection among neonates. Annals of International Medical and Dental Research, 2: 5-8.

16. Nazir A (2019): Multidrug-resistant Acinetobacter septicemia in neonates: A study from a teaching hospital of Northern India. Journal of Laboratory Physicians, 11(1): 23-28.

17. Hannan A, Qamar M, Usman M et al. (2013): Multidrug resistant microorganisms causing neonatal septicemia: In a tertiary care hospital Lahore, Pakistan. African Journal of Microbiology Research, 7(19): 1896-1902.

18. Akter M, Jahan N, Islam M et al. (2015): Multidrug resistant Acinetobacter spp. Blood stream infection in a neonatal intensive care unit of an urban specialized hospital in Dhaka. Journal of Dhaka Medical College, 24(1): 47-52.

19. Awad H, Mohamed M, Badran N et al. (2016): Multidrug-resistant organisms in neonatal sepsis in two tertiary neonatal ICUs, Egypt. Journal of the Egyptian Public Health Association, 91(1): 31-38.

20. Hsu J, Chu S, Lien R et al. (2014): Case-control analysis of endemic Acinetobacter baumannii bacteremia in the neonatal intensive care unit. American Journal of Infection Control, 42(1): 23-27.

21. Yusef D, Shalakhti T, Awad S et al. (2018): Clinical characteristics and epidemiology of sepsis in the neonatal intensive care unit in the era of multi-drug resistant organisms: a retrospective review. Pediatrics \& Neonatology, 59(1): 35-41. 